\title{
Propuesta de un Modelo Integral de Accountability para la Educación Superior en Chile
} PROPOSAL OF AN INTEGRAL MODEL OF ACCOUNTABILITY FOR HIGHER EDUCATION IN CHILE

Guillermo M. Riquelme Silva', Alberto A. López Toro², Antonio M. Ciruela Lorenzo ${ }^{2}$

1. Autónoma University of Chile

2. University of Málaga, Spain

\section{RESUMEN}

Depositarias de la confianza de la ciudadanía y del Estado, las instituciones de educación superior han desarrollado su misión de formación e investigación dentro de un contexto acotado. En la actualidad, hay una demanda de parte del Estado y la ciudadanía de una mayor participación y supervisión del sistema de educación superior, para la mejora de la calidad de la educación impartida, de una parte, y, de otra, para dar cuenta de los recursos públicos invertidos en educación superior. En este contexto, este trabajo propone un modelo integral de rendición de cuentas para la educación superior en Chile. Su objetivo es proponer dicho modelo como herramienta de evaluación de las políticas públicas en educación superior, para que esta contribuya productivamente a las actividades económicas del país. Como pondremos de manifiesto en el trabajo, la rendición de cuentas no sólo se limita al aspecto financiero, sino que incorpora muchas otros aspectos tales como la autoridad y gobierno, la responsabilidad social, la democracia y la participación, la probidad y la transparencia informativa, la política y la ética, entre otras.

(Riquelme G, López A, Ciruela A, 2018. Propuesta de un Modelo Integral de Accountability para la Educación Superior en Chile. Cienc Trab. May-Ago; 20 [62]: 107-115).

Palabras Clave: GESTIÓN DE LA CALIDAD, EDUCACIÓN SUPERIOR, RENDICIÓN DE CUENTAS, GESTIÓN DE LA EDUCACIÓN, POLÍTICA DE EDUCACIÓN SUPERIOR.

\section{ABSTRACT}

Depositary trust of citizens and the state, higher education institutions have developed their mission training and research within a limited context. At present, there is a demand from the state and citizens of increased participation and supervision system of higher education, to improve the quality of education provided in one hand and another to account for public funds invested in higher education. In this context, this paper proposes a comprehensive model of accountability for higher education in Chile. Its purpose is to propose such a model as a tool for assessing public policies in higher education, for higher education to contribute productively to the economic activities of the country. As we will show at work, accountability is not only limited to the financial aspect, but incorporates many other aspects such as the authority and governance, social responsibility, democracy and participation, probity and transparency of information, politics and ethics, among others.

Key Words: QUALITY MANAGEMENT, HIGHER EDUCATION, ACCOUNTABILITY, EDUCATION MANAGEMENT, HIGHER EDUCATION POLICY.

\section{INTRODUCCIÓN}

Las instituciones de educación superior históricamente habían sido depositarias de la confianza de la ciudadanía y de la fe pública del Estado para la formación y graduación del capital humano avan-

Correspondencia / Correspondence:

Guillermo Riquelme Silva

e-mail: griquelmes@uautomoma.cl

Alberto A. López Toro.

e-mail: aalopez@uma.es

Antonio M. Ciruela Lorenzo

e-mail: acl@uma.es

Recibido: 23 de marzo de 2018 / Aceptado: 10 de Junio de 2018 zado, dentro de un contexto acotado de participantes de la población en el sistema de educación superior. No obstante, en la década de los 80' del siglo pasado, los países desarrollados comenzaron a implementar políticas públicas de mayor cobertura al acceso a la educación superior, lo cual posibilitó un gran crecimiento de las matrículas en las diversas instituciones de educación superior, tanto públicas como privadas, cuestionándose paralelamente su calidad. Es así como Alexander ${ }^{1}$ plantea que la pérdida de credibilidad en la autorregulación de las Instituciones de Educación Superior (IES) ha presionado a los Estados a exigir a las universidades a dar cuenta de los resultados de su gestión. Es más, se plantea la creación de nuevas formas de medir el rendimiento y la productividad de los recursos que los Estados invierten en educación superior. Pasando desde una evaluación institucional con pares evaluadores, a una evaluación práctica con enfoque económico utilitarista asociado a las necesidades actuales de la economía mundial. 
En este nuevo escenario se demandará de parte del Estado, de una $\mathrm{u}$ otra forma, una mayor participación y supervisión del sistema de educación superior, persiguiendo, por un lado, la mejora de la calidad de la educación impartida ${ }^{2} \mathrm{y}$, por otro, la redituabilidad de los recursos públicos invertidos en educación superior. Tales demandas y su creciente nivel de exigencia requieren de un fuerte y holístico Sistema de Rendición de Cuentas que, sin duda, comporta un gran desafío de Política y Gestión Educativa a nivel de Estado, Sistema Educativo e Instituciones Educativas. ${ }^{3}$

En este contexto, el presente trabajo* pretende ser una aproximación a un modelo integral de rendición de cuentas para la educación superior en Chile, en particular, y de las universidades del mundo, en general. Que permita una adecuada toma de decisiones en el espectro de las políticas públicas en educación superior, ya que sería muy lamentable que el aumento evidenciado en el mundo de la producción de titulados y/o graduados no haya sido acompañado de un riguroso control de calidad asociado a la medición de los aprendizajes y competencias técnicas y profesionales necesarias para aportar productivamente a las diversas actividades económicas del país. Esto causaría en el largo plazo un daño al Estado, a los estudiantes, a sus familias, a las empresas y a toda la sociedad en su conjunto. ${ }^{4}$

\section{MARCO CONCEPTUAL}

Para Hendel y Lewis ${ }^{5}$, los países en transición, como es el caso de Chile, han adoptado la acreditación como una herramienta de política pública en educación para la mejora de la cuestionada calidad de la educación. No obstante, parece insuficiente al alero de lo evidenciado por los países desarrollados, debiéndose converger desde una evaluación hacia una rendición de cuentas, sin por ello excluir los procesos de evaluación y acreditación. Todos ellos son parte fundamental del sistema de aseguramiento de la calidad. Es más, se plantea que la acreditación debería permitir la equivalencia de los títulos a nivel internacional, lo que tiene mucho sentido con la estandarización curricular. ${ }^{6}$

La rendición de cuentas por el uso de los recursos financieros, fundamentalmente públicos, se convierte en una exigencia cada vez mayor en todos los países del mundo, en especial, en aquellos que han ido aumentado significativamente los aportes públicos al sistema educativo. Junto con ello, el Estado debe garantizar que las instituciones educativas cumplan los fines para los cuales fueron creadas, supervisando su gestión tanto institucional como docente, sin con ello afectar la libertad académica que les es propia como práctica educativa.

La rendición de cuentas, a diferencia de la acreditación, no contempla la participación de pares evaluadores externos, que ha sido tradicionalmente parte del proceso de evaluación y acreditación institucional en países desarrollados como Estados Unidos, Reino Unido y también en países emergentes como Chile.

La rendición de cuentas obliga a todas las instituciones educativas a hacerse responsable de los resultados formativos y que su valor agregado sea coherente con la inversión de recursos públicos destinados a tales instituciones. Para David A. Shupe ${ }^{7}$, la rendición de cuentas en educación superior implica que una persona y/o institución es responsable ante otra persona y/o institución por sus

\footnotetext{
* Este trabajo es parte de la Tesis doctoral: Estudio de la Accountability en la educación superior en Chile, entre los años 1990-2009 (Riquelme, 2014).
}

procesos y resultados, dada la transferencia de cierta cantidad de recursos. Es así como una parte es responsable de la productividad de un proceso y la calidad de un producto, y la otra parte es la que evalúa el resultado. Para clarificar lo anterior, Shupe utiliza distintos diagramas de flujo a fin de dar cuenta de la accountability en la educación superior.

En primer lugar, se analiza en general el sistema de accountability, en donde se incorpora desde el lenguaje de los negocios al productor, el proceso productivo, el producto propiamente dicho y, finalmente, el consumidor o cliente, debiendo el productor dar cuenta de los resultados al consumidor que es quien finalmente evalúa la calidad del producto (Ver Figura 1).

Figura 1.

Sistema General de Accountability. PRODUCTIVIDAD CALIDAD

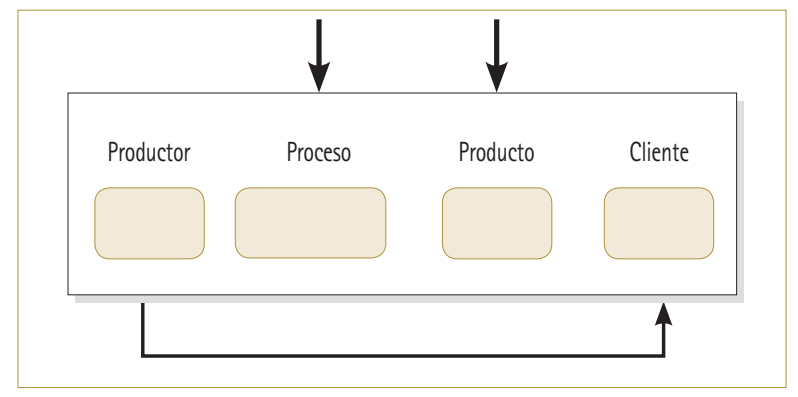

Fuente: David A. Shupe (1999).

Según Shupe ${ }^{7}$, este sistema general de accountability puede adoptar distintos enfoques o miradas para cada uno de sus componentes:

$1^{\circ}$ Enfoque: Proceso enseñanza-aprendizaje que contempla al estudiante como el generador de su conocimiento, mediante la comprensión de los contenidos de los cursos, obteniéndose como producto cierto tipo de trabajos desarrollados por el estudiante, siendo el instructor y/o profesor quien finalmente debe evaluar el resultado.

$2^{\circ}$ Enfoque: Proceso enseñanza-aprendizaje que contempla a los planes y programas de estudio como los componentes generadores de conocimiento, mediante la aplicación del diseño curricular en cuanto a los contenidos de los cursos, obteniéndose como producto la certificación de títulos y/o grados. Siendo el estudiante quien finalmente debe evaluar el resultado.

$3^{\circ}$ Enfoque: Proceso enseñanza-aprendizaje que contempla a las instituciones educativas como las productoras del conocimiento, mediante la preparación de los estudiantes para desempeñarse en el trabajo e integrarse productivamente a la sociedad, obteniéndose como producto formal estudiantes calificados para el trabajo y la vida en sociedad. Siendo los empleadores y la comunidad la que finalmente debe evaluar el producto final.

Tanto el proceso centrado en los planes y programas como el proceso centrado en las instituciones educativas convergen hacia una lógica de mercado donde el cliente (estudiantes-comunidadempleadores) es el que finalmente evalúa los resultados del proceso. En tal sentido, los procesos de acreditación que se focalizan en la evaluación de la calidad de las instituciones educativas y su respectiva oferta académica han adoptado en parte la lógica del mercado, en donde los procesos documentados se superponen a la real búsqueda de la calidad de la enseñanza. ${ }^{8}$ 
La certificación de la calidad a través de la acreditación en países desarrollados como EE.UU., Reino Unido y también países emergentes como Chile han incorporado desde la Gestión de la Calidad Total (TQM) un enfoque centrado en eficiencia y eficacia del negocio, en donde los estudiantes se constituyen como los consumidores y el carácter de bien público de la educación se pierde y se transforma en un bien privado. ${ }^{9}$

También se puede hablar de la "Rendición de Cuentas de Mercado", que según Thaddeus Metz ${ }^{10}$ está asociada a la elección informada que deben realizar los estudiantes en cuanto a qué institución de educación superior van a ingresar. Por tanto, se alude al gobierno con el deber de informar respecto de la calidad de las instituciones de educación superior. Además, existen desacuerdos en torno a lo que debe ser una educación de calidad en la propia universidad, en el gobierno y en las instituciones públicas. ${ }^{11}$ Sin embargo, parece insuficiente solo tener el deber de informar; por tanto, se discuten otros tipos de rendición de cuentas.

Por su parte, Zapata y Fleet ${ }^{12}$ constatan la insuficiente información existente sobre las instituciones de educación superior y plantean que el predominio de indicadores de calidad institucional relacionados con las universidades de excelencia incentiva una mayor provisión de información entre este tipo de universidades, pero inhibe la rendición de cuentas entre las universidades de menor excelencia y bajo prestigio acumulado.

En términos generales, Metz ${ }^{10}$ propone un marco analítico para la rendición de cuentas, que ha sido resumido y adaptado a los fines del presente estudio de la siguiente manera:

"Existe un determinado agente (el gobierno) que tiene el deber de proporcionar información a otro agente (estudiantes-empleadorescomunidad) de una forma determinada sobre el grado en que el agente de educación superior (universidad) ha cumplido con sus deberes (misión, visión, proyecto educativo) por el bien de la sociedad toda, tomando las medidas pertinentes para el aseguramiento de la calidad".

De este marco analítico cabe destacar la diferencia entre el deber que tiene el gobierno respecto de informar, y el deber que tiene respecto del bienestar de la sociedad toda. Esto es muy relevante, ya que en la discusión de la responsabilidad muchas veces queremos saber, por un lado, lo que una universidad debe hacer $y$, por otro, el propósito final para hacerlo.

Por ejemplo, se podría pensar que la universidad tiene el deber de fomentar las carreras de ingeniería y tener la mayor matrícula posible en esta área del conocimiento. Esto, con el fin de mejorar la calificación de los profesionales para el desarrollo de proyectos de ingeniería e infraestructura productiva del país, que posibilitaría un mayor crecimiento económico de la nación.

Ahora, emerge también del análisis de la rendición de cuentas la obligación o deber del gobierno de responder por las fallas que se produzcan en el sistema educativo, fundamentalmente, en el resultado esperado por la sociedad. En este caso se incorpora a la rendición de cuentas el concepto de "pedir cuentas", que se haría efectivo en la medida que la población se empodere de sus derechos y atributos como ciudadano.

Por ejemplo, en Brasil existe el Examen Nacional de Cursos (ENC) que representa un examen anual obligatorio para cada estudiante de último año de la universidad en una lista específica de las disciplinas, lo cual forma parte de un sistema de rendición de cuentas que premia y penaliza las universidades en función de su rendimiento. Instituciones con buenos resultados en el ENC pueden inscribir más alumnos, además de abrir nuevos campus. En cambio, a aquellas instituciones con malos resultados se les prohíbe temporalmente la admisión de nuevos alumnos y no pueden abrir nuevos campus. Es decir, se aplicaban sanciones que están ciertamente en la lógica de responder a la ciudadanía en cuanto a "pedir cuentas". No obstante, las instituciones afectadas recurren a los tribunales de justicia y consiguen revertir las sanciones, con lo cual la medida instaurada por el gobierno no ha sido completamente eficaz. Sin embargo, los resultados del ENC son publicados por el gobierno y afectan negativamente a las instituciones con malos resultados y benefician a las instituciones con buenos resultados, en cuanto a que los alumnos optan por estas últimas al momento de matricularse.

Sin duda, que todo esto se establece con el fin de generar los estímulos para mejorar la calidad de la educación en un mercado educacional cada vez más liberalizado. En donde los estudiantes podrian mostrar sus puntuaciones al solicitar empleo y además acceder a becas públicas de especialidad y postgrado.

Otros antecedentes se obtienen de Huisman y Currier ${ }^{13}$, en un estudio sobre rendición de cuentas en 4 universidades de Francia (Universidad de Avignon), Países Bajos (Universidad de Twente), Noruega (University of Oslo) y Estados Unidos (Boston College). En tal estudio se señala que los gobiernos abogan por medidas de rendición de cuentas, pero luego se abstienen de hacer cumplir los instrumentos de política específicos. En realidad, es difícil para ellos vigilar si las instituciones de educación superior están cumpliendo cabalmente con su rol educador y lo establecido en su misión, visión y proyecto educativo.

Además, se distingue un patrón en común, esto es, el énfasis en la evaluación de los procesos, más que en los resultados, y las sanciones raramente son aplicadas. Lo cual también es común en algunos países emergentes como Chile.

En los resultados finales del informe, se destaca que casi la mitad de las respuestas indicaron que había una falta de mecanismos de rendición de cuentas y que más bien existían los tradicionales mecanismos de control asociado, por ejemplo, al número de estudiantes, sus grados de deserción y aprobación. También se señala que en torno a la mitad de las respuestas indican que existe una rendición de cuentas "hacia fuera", y no tenían ningún efecto inmediato en la calidad de la educación dentro de la institución. ${ }^{13}$ En este contexto, tal como lo señalan Conner y Rabovscky ${ }^{14}$, los estudios e investigaciones respecto de la interacción entre las variables de políticas estatales y los resultados institucionales es un terreno particularmente fértil en el segmento de la educación superior, especialmente teniendo en cuenta las recientes discusiones de políticas de rendición de cuentas y la lucha entre las mejoras de ingeniería estatal en el desempeño institucional y la preservación de la autonomía institucional en la educación superior.

Más aun, cuando se ha evidenciado que el financiamiento de la educación superior en conjunto con otros instrumentos de políticas públicas son relevantes en cuanto a las formas de abordar la desigualdad respecto del acceso y equidad de la educación superior. Para el caso de Chile, el financiamiento de la educación superior está en pleno auge y se espera continúe creciendo, por lo cual se hace notable como línea de investigación en cuanto al impacto en la desigualdad y equidad en el acceso a la educación superior, y su contribución al progreso social.

En este contexto de rendición de cuentas integral, el informe sobre la Renovación de los Sistemas de Rendición de Cuentas de la Educación Superior ${ }^{15}$ propone un marco general para los Estados Unidos. Este marco sirve como guía para la visualización y análisis de un Sistema 
de Rendición de Cuentas Integral. Su estructura se representa en forma de pirámide, donde en su base se encuentran las diversas instituciones de educación superior, quienes deben proporcionar los informes y análisis detallado de la actividad educativa (por ejemplo, cantidad de estudiantes, número de titulados, número de deserciones, etc.). A nivel intermedio de la pirámide se encontraría todo el sistema educativo, quienes deben proporcionar los informes y análisis a nivel agregado (por ejemplo, el total de carreras por área del conocimiento, total de egresados y titulados en diferentes áreas, etc.). Finalmente, en la cúpula de la pirámide se encontraría el Estado, centrado en cuestiones macro de política educativa y su impacto en la economía nacional (por ejemplo, el rendimiento del sistema en el cumplimiento de necesidades económicas del país, retorno de la inversión, etc.). La Figura 2 muestra el modelo descrito de sistema de rendición de cuentas integral.

De la Figura 2 se puede observar que a nivel del Estado la atención está sobre el logro o cumplimiento de los objetivos del sistema educativo, el satisfacer los requerimientos de mano de obra calificada y, consecuentemente con ello, contribuir al aumento de la productividad. En cambio, a nivel del sistema educativo, el interés se centra en la tasa de terminación o finalización de los estudios (egresados y/o titulados), las mejoras de las instituciones y sus diversas escuelas y departamentos, reducción de la deserción y reducción del costo de terminación de estudios, además de determinar los programas de estudios pertinentes y su atingencia a las diversas instituciones. Por último, en la base de la pirámide la preocupación está en el acceso a las instituciones educativas (admisión), el éxito de programas remediales, tasa de aprobación de cursos y reducir el tiempo y los créditos de aprobación de los cursos, entre otros.

En América Latina un aporte significativo en la formulación de modelos de rendición de cuentas en educación lo ha realizado el Programa para la Reforma Educacional en América Latina. La PREAL finalmente ha propuesto seis variables de medición de la Accountability para los países de América Latina: ${ }^{16}$

(a) Estándares; (b) Información; (c) Consecuencias; (d) Autoridad; (e) Capacidades; y, (f) Comunicación.

El principal objetivo de la PREAL al identificar estas variables es mejorar la calidad de la educación en América Latina, y contempla como supuestos básicos:

1. Que las personas, en particular los niños, tienen la capacidad de aprender si se les provee educación de buena calidad ajustada a sus necesidades, independientemente de su etnia, género o nivel socioeconómico, entendiendo, además, que recibir educación de calidad es un derecho.

2. Se considera que los maestros, directores de escuela y funcionarios del sector educativo están motivados no solo a hacer bien su trabajo y servir a los alumnos a su cuidado, sino también a procurarse una vida lo más fácil y cómoda posible, dada la tendencia natural a buscar su propia comodidad (lo que los economistas llaman "maximizar el bienestar").

3. La información basada en pruebas es deseable para la Accountability, pero que las pruebas no son la única fuente de información y ni siquiera constituyen un ingrediente esencial para un sistema de la Accountability.

Cabe destacar que este modelo y, por ende, sus supuestos están orientados específicamente a la educación escolar. Sin embargo, consideramos totalmente extrapolable dichas variables a la rendición de cuentas en la Educación Superior. Fundamentalmente, porque son propuestas desde la mirada macro de las políticas públicas.

Figura 2. Modelo Integral de Rendición de Cuentas.

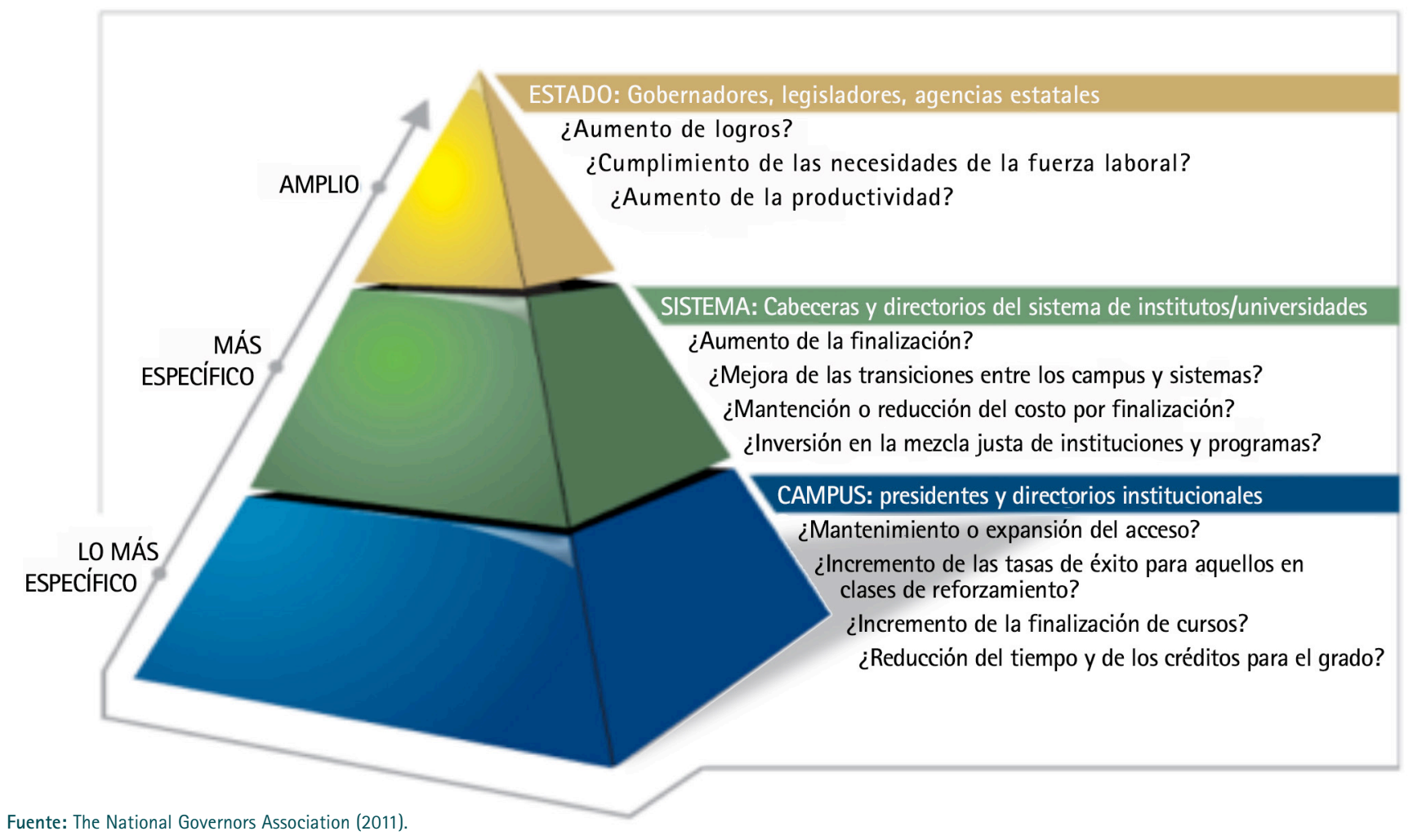




\section{METODOLOGÍA}

En razón de todos los antecedentes teóricos expuestos y desde la mirada macro de política en educación superior, se ha procedido a distinguir las principales variables que permitirian rendir cuentas en educación superior y que cubren de mejor manera el espectro de requerimientos de información de los distintos agentes intervinientes de la sociedad toda. En este aspecto nos referimos a una rendición de cuentas integral.

Es así como podemos rescatar de los análisis y conceptualizaciones teóricas los principales aspectos que se deben contemplar en una rendición de cuentas en educación superior, desde un enfoque macro de política educacional y de carácter social.

\section{Entre los aspectos más relevantes se encuentran:}

- La calidad debe ser medida; para ello se debe evaluar tanto el proceso de enseñanza como el de aprendizaje. Siendo este último el más relevante en cuanto a los resultados esperados por la sociedad. Al respecto deben existir pruebas o exámenes habilitantes.

- Información pública, completa, transparente, oportuna y pertinente para la toma de decisiones de los diversos agentes de la educación superior. A través de métricas en educación superior que aborden no solo el proceso de enseñanza-aprendizaje, sino también la gestión institucional y la redituabilidad financiera.

- Que existan medidas y/o sanciones para quienes no estén cumpliendo con sus proyectos educativos y falten a la fe pública.

- Se requiere de una autoridad reguladora y responsable ante la sociedad toda que, sin duda, debe ser parte del Estado. Donde la autoridad se ejerza desde la técnica para la permanente mejora de la calidad.

- Se debe contar con una capacidad técnica a todo nivel de la pirámide de la estructura de rendición de cuentas.

- Se requiere, como complemento, de un enfoque económico utilitarista asociado a las necesidades de la economía: formación de capital humano avanzado, productividad y remuneraciones.

- La rendición de cuentas debe ser institucionalizada a nivel de estado y comunicada en sus diferentes niveles: estado, sistema educativo e instituciones educativas.

- Debe permitir estudiar a nivel macro el sistema educacional de un país.

- Se debe focalizar en la política educacional de los gobiernos.

- Identificar las variables claves de medición de la accountability educacional.

- Énfasis en la responsabilidad y rendición de cuentas de los gobiernos.

- Propósito principal: mejorar la calidad de la educación.

En consideración a que las variables de medición de la accountability educacional planteadas por la PREAL se ajustan notablemente al contexto de la accountability en educación superior, será considerado como base para la propuesta de un Modelo Integral de Accountability para la Educación Superior en Chile. No obstante, se agregará una variable adicional al modelo de la PREAL, denominada; "Resultados". Con ella se pretende complementar y profundizar el modelo de rendición de cuentas en términos del gasto público en educación y la redituabilidad de esos recursos para la sociedad, en términos de capital humano avanzado, empleo calificado, productividad y remuneraciones.

En este contexto, se propone un modelo de acercamiento sisté- mico y con una bajada a lo operacional, como un aporte al desafío de dar un uso y aplicación al diseño, la ejecución y, fundamentalmente, a la Evaluación de las Políticas Públicas en Educación Superior. En este afán, se utiliza como referencia el "Modelo Chileno de Gestión de Excelencia"17, que a juicio de los investigadores, permitiría sistematizar la evaluación de las variables de la accountability, así como instalar en el sistema de educación superior una cultura hacia la mejora continua de las variables críticas de gestión, como son: la estructura organizacional, los procesos de gestión educativa, el liderazgo, la relación con la comunidad educativa y la responsabilidad social.

\section{RESULTADOS}

En términos generales, el modelo contempla las 7 variables asociadas a la Accountability en la Educación Superior. Todas estas variables fueron descritas, analizadas y contrastadas con los antecedentes empíricos recabados en la presente investigación, y que dieron cuenta del nivel de la Rendición de Cuentas en la Educación Superior en Chile: Estándares, Capacidades, Autoridad, Resultados, Información, Consecuencias y Comunicación.

Las siete variables en cuestión se interrelacionan y contribuyen conjuntamente a la rendición de cuentas (Figura 3). Es decir, cada una de ellas entrega y recibe de las otras, insumos de evaluación que permiten finalmente entregar una rendición de cuentas integral. Convergiendo a dos ámbitos de evaluación:

a) Gestión Educacional y,

b) Calidad de la Educación

Las variables y sus interrelaciones se pueden explicar de la siguiente manera:

1. La accountability requiere, como parámetro de medición fundamental, el diseño del proceso formativo que representa finalmente el quehacer de las instituciones educativas. Este diseño formativo es el denominado diseño curricular, el cual debe contener los planes y programas de estudio que, entre otras cosas, definan la cantidad de horas de la asignatura, la cantidad de horas semanales, los objetivos a alcanzar, la didáctica a utilizar, la forma de evaluación y los requisitos de aprobación. Para ser medido, el currículo debe cumplir con ciertos estándares de comparación. Para el caso de Chile estos estándares pueden ser asumidos como el Sistema Créditos Transferibles en Chile (SCT-Chile), que ya se vienen utilizando por algunas universidades. Se hace necesario entonces, que a nivel de Política Educacional se implemente el SCT-Chile para todo el sistema de educación superior. ${ }^{18}$

2. Las competencias de las instituciones y sus académicos deben dar cuenta de las exigencias impuestas por el currículo y la evolución que este experimente acorde al desarrollo del conocimiento. Debiéndose implementar en las instituciones educativas una política institucional de desarrollo de capacidades, como parte integral de su quehacer educacional. De manera tal que puedan ser los llamados a aportar en los cambios e innovaciones curriculares, que luego serán integrados en el SCT-Chile, reformulándose los estándares.

3. La autoridad debe cumplir con abarcar la transversalidad de la comunidad educativa, esto es, ostentar una representatividad de todos los segmentos y grupos de interés, que afectan y son afectados por el proceso educativo. Esto puede ser plasmado a través de un sistema democrático de gobierno universitario en 
Figura 3. Esquema General del Modelo de Rendición de Cuentas para la Educación Superior.

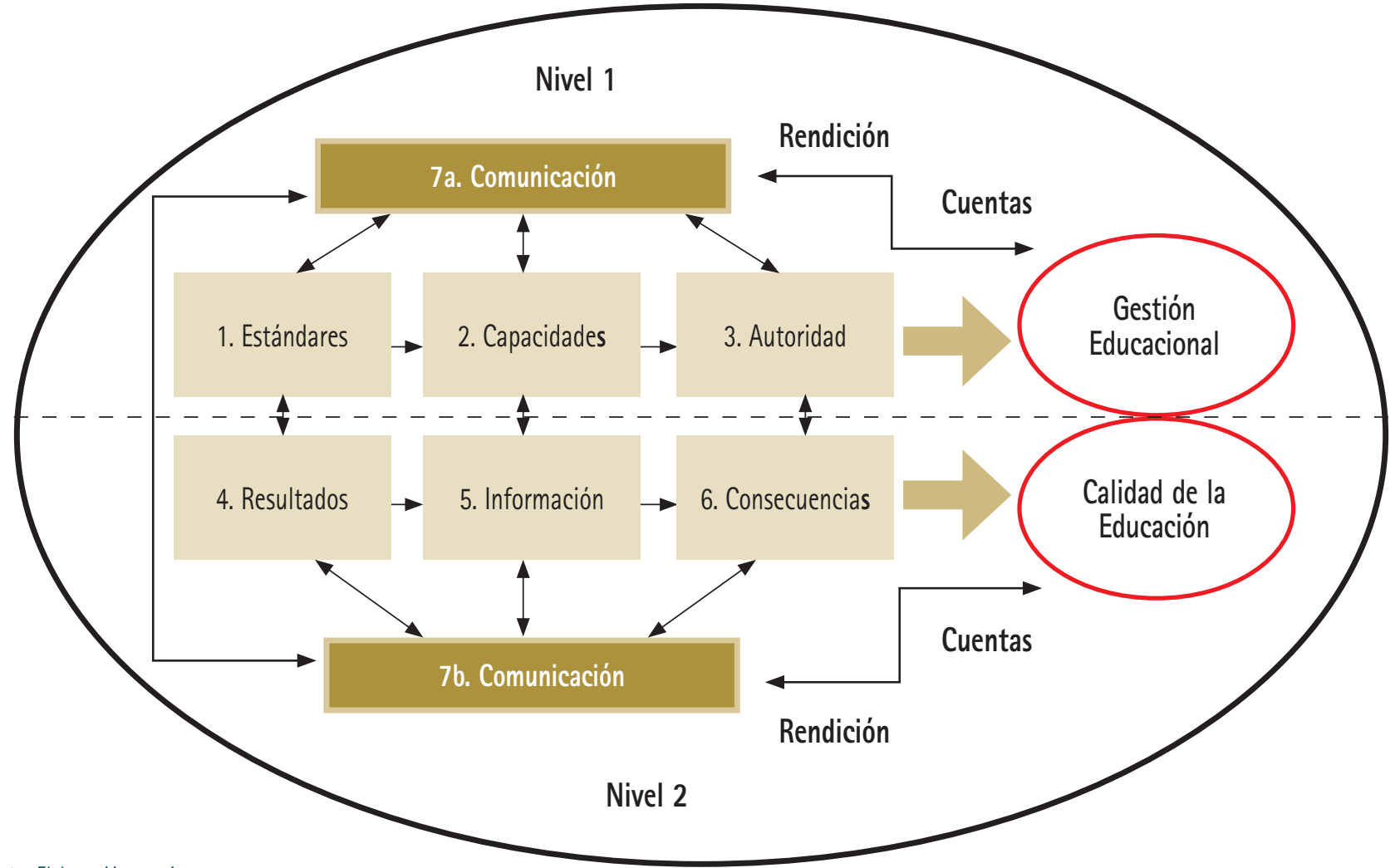

Fuente: Elaboración propia.

donde se ejerza el derecho de voz y voto de los académicos, funcionarios, estudiantes y la comunidad educativa. El hablar con autoridad dependerá fundamentalmente del aporte que cada grupo realice al proyecto educativo en el que participa, obligándose a desarrollar las capacidades que les permitan contribuir al proceso educativo.

4. Los resultados deben dar cuenta, por un lado, de los resultados de los aprendizajes esperados del proceso formativo. Esto, a través de la aplicación de pruebas nacionales de medición, en distintos niveles de la carrera, incluyendo un examen final que podría ser habilitante para el ejercicio profesional. Además de la evaluación permanente de los profesionales que en el ejercicio de su profesión deben actualizar continuamente sus conocimientos $\mathrm{y}$, por otro, de la redituabilidad de los recursos económicos y financieros destinados a la educación. De forma tal de evaluar el impacto en el bienestar de la propia comunidad educativa y de la sociedad toda. Los elementos objetivables en tal sentido serán: la formación de capital humano, su aporte a la productividad del país y su consecuente nivel de remuneraciones.

5. La información debe perseguir, como objetivo final, la difusión a todo el sistema educativo y a sus grupos de interés de todos los resultados identificados en la variable Resultados. Esto es, tanto información económica-financiera como información de los logros de aprendizaje y competencia profesional. Esta información permitirá retroalimentar a todas las variables de la accountability.

6. Las consecuencias deben ser, por un lado, estímulos positivos para premiar y hacer visible a todo el sistema de educación superior las prácticas que son deseables en el quehacer educacional y, por otro, sanciones ejemplificadoras para aquellas actuaciones $u$ omisiones que pongan en riesgo el devenir educativo y perjudique el proceso de mejora continua que se pretende instalar. Las consecuencias, sin duda, no pueden ser dictaminadas sin un adecuado nivel de información que proporcione los antecedentes necesarios y suficientes para la toma de decisiones.

7. La última variable, comunicación, afecta transversalmente a todas las demás variables, ya que se sustenta de ellas para proporcionar un mensaje claro y convincente de los atributos y beneficios del modelo de accountability. Propendiendo a que la ciudadanía se integre y se sienta parte de la mejora continua del sistema de educación superior en Chile. Canalizando su mensaje a través de 2 grandes vías; la Gestión Educacional (7a) y la Calidad de la Educación (7b).

Para que el modelo propuesto pueda llevarse a la práctica, se hace necesario diseñar una estructura de evaluación para los dos ámbitos de rendición de cuentas: la Gestión Educacional y la Calidad de la Educación. Lo cual implica recoger para cada ámbito de rendición de cuentas las variables de evaluación que la sustentan. En este último aspecto se dará una bajada operacional al modelo propuesto a través de la operacionalización de tales variables, identificando para cada una de ellas algunos Criterios de Evaluación que, a su vez, tendrán asociados determinados Elementos de Evaluación.

Serán considerados como criterios de evaluación los principales aspectos que dan cuenta y caracterizan a cada una de las variables del modelo. Por tanto, los criterios de evaluación permiten visualizar en forma desagregada el significado de la variable. 
Tabla 1.

Operacionalización de la variable Estándares.

\begin{tabular}{|c|c|c|}
\hline VARIABLE & CRITERIOS & ELEMENTOS DE EVALUACIÓN \\
\hline \multirow[t]{2}{*}{ Estándares } & $\begin{array}{l}\text { Desarrollo } \\
\text { de Estándares }\end{array}$ & $\begin{array}{l}\text { Adopción del Sistema de Créditos Transferibles. (SCT-Chile). } \\
\text { Planificación del Proceso de Conversión al SCT-Chile. } \\
\text { Programas Académicos Involucrados en el SCT-Chile. } \\
\text { Determinación de Carga Horaria Anual bajo el SCT-Chile } \\
\text { por Programa Académico. } \\
\text { Determinación por Programa Académico de Carga } \\
\text { Horaria Semanal bajo el SCT-Chile. } \\
\text { Determinación de Carga Académica Semanal por } \\
\text { Asignatura. }\end{array}$ \\
\hline & $\begin{array}{l}\text { Implementación } \\
\text { de Estándares }\end{array}$ & $\begin{array}{l}\text { Difusión y sociabilización del SCT-Chile Adoptado por } \\
\text { la Institucional Educacional. } \\
\text { Ejecución de los Planes y Acciones de Conversión al } \\
\text { SCT-Chile. } \\
\text { Niveles de Avance de la Ejecución de lo Planificado. } \\
\text { Programas Académicos con Implementación Completa } \\
\text { del SCT-Chile. }\end{array}$ \\
\hline
\end{tabular}

Tabla 2.

Operacionalización de la variable Capacidades.

\begin{tabular}{|c|c|c|}
\hline VARIABLE & CRITERIOS & ELEMENTOS DE EVALUACIÓN \\
\hline \multirow{3}{*}{ Capacidades } & $\begin{array}{l}\text { Gestión del } \\
\text { Personal }\end{array}$ & $\begin{array}{l}\text { Políticas de Selección del Personal Académico. } \\
\text { Difusión y Reclutamiento del Personal Académico. } \\
\text { Estructura Organizacional y Administración del } \\
\text { Trabajo Académico. }\end{array}$ \\
\hline & $\begin{array}{l}\text { Desempeño y } \\
\text { Reconocimiento } \\
\text { de las Personas }\end{array}$ & $\begin{array}{l}\text { Evaluación del Desempeño del Personal Académico. } \\
\text { Identificación de la Necesidades de Nuevas } \\
\text { Capacidades Académicas. } \\
\text { Reconocimiento al Desarrollo de las Capacidades } \\
\text { Académicas. } \\
\text { Compromiso a Nivel Académico, Institucional y } \\
\text { Gubernamental para el Desarrollo de Capacidades. }\end{array}$ \\
\hline & $\begin{array}{l}\text { Formación y } \\
\text { Desarrollo de } \\
\text { Capacidades }\end{array}$ & Nivel de Especialistas, Investigadores y Científicos. \\
\hline
\end{tabular}

Por su parte, los elementos de evaluación representan para cada criterio de evaluación indicadores específicos de la presencia y el nivel alcanzado de aquello que se establece en cada criterio de evaluación.

El diseño de la Estructura de Evaluación contempla, básicamente, 7 variables de rendición de cuentas. Luego, todas estas variables serán explicadas a través de 21 criterios de evaluación. Los que a su vez serán operacionalizados con la descripción de 75 elementos de evaluación. En particular, la Rendición de Cuentas de la Gestión Educacional para sus tres variables -Estándares, Capacidades y Autoridad-, tendrá 8 criterios de evaluación que luego se operacionalizan a través de 29 elementos de evaluación.

Por su parte, para la Rendición de Cuentas de la Calidad de la Educación, en cuanto a sus tres variables -Resultados, Información y Consecuencias-, se tendrán 10 criterios de evaluación, que luego se operacionalizan a través de 37 elementos de evaluación.

Finalmente, la variable Comunicación, que es transversal a ambos ámbitos de la rendición de cuentas, tendrá 3 criterios de evaluación, que se operacionalizan a través de 9 elementos de evaluación.
Tabla 3.

Operacionalización de la variable Autoridad.

\begin{tabular}{|l|l|l|}
\hline VARIABLE & CRITERIOS & ELEMENTOS DE EVALUACIÓN \\
Liderazgo y & $\begin{array}{l}\text { Ascendencia y Disposición de las Autoridades para la } \\
\text { Institucional } \\
\text { Mejora Continua. } \\
\text { Catoridad } \\
\text { Capacidad de Convocatoria para Abordar los Procesos } \\
\text { de Mejora Continua. } \\
\text { Estilos de Dirección Participativos y Democráticos. } \\
\text { Altos Niveles de Competencias Técnicas de las } \\
\text { Autoridades. } \\
\text { Autoridad } \\
\text { Colegiada }\end{array}$ & $\begin{array}{l}\text { Presencia de Gobiernos Universitarios con Alta } \\
\text { Representatividad. } \\
\text { Representación en Comisiones Gubernamentales; Desarrollo } \\
\text { de Politicas en Educación Superior. } \\
\text { Control Gubernamental y Supervisión Técnica del Proceso } \\
\text { Formativo y de la Gestión Educativa. } \\
\text { Representación Estudiantil; Centros de Alumnos y } \\
\text { Federaciones de Estudiantes. Participación en } \\
\text { Gobierno Universitario. } \\
\text { Representación Familias, Centros de Padres y Tutores. } \\
\text { Participación en Gobierno Universitario. } \\
\text { Representación Comunidad: Organizaciones Comunitarias } \\
\text { Comunidad } \\
\text { Educativa su Participación en Gobierno Universitario. }\end{array}$ \\
&
\end{tabular}

\section{REFLEXIONES FINALES}

A nivel general, la accountability se presenta como una rendición de cuentas que no sólo se limita al aspecto financiero, sino que incorpora muchas otros aspectos tales como la autoridad y gobierno, la responsabilidad social, la democracia y la participación, la probidad y la transparencia informativa, la política y la ética, entre otras.19 Además, la accountability en educación implica asumir la responsabilidad de las propias acciones y sus resultados, lo que hace que pueda ser considerada como una herramienta para la evaluación de la gestión y política pública en educación16 y, por ende, en la educación superior. Como apunta dicho autor, la accountability contribuye a la mejora de la calidad en la educación y se percibe como un mecanismo para la mejora de la equidad en la educación.

Como fue señalado en la introducción de la presente propuesta de Modelo de Accountability para la Educación Superior, se pretende proporcionar una visión holística de la Rendición de Cuentas en la Educación Superior en Chile, identificándose dos grandes ámbitos de evaluación de la política de educación superior, esto es, la Gestión Educacional y la Calidad de la Educación. De forma tal de servir como orientación preliminar para la discusión, análisis y mejora de las políticas públicas en educación superior. Por tanto, se debe tomar como un documento de trabajo, en donde los distintos actores involucrados en la educación superior en Chile participen en su mejora continua, orientando la planificación, organización $\mathrm{y}$, principalmente, la evaluación de las políticas públicas en educación superior.

En cuanto a la estructura del modelo, faltaría por incorporar los Indicadores de Evaluación para cada uno de los Elementos de Evaluación. Dichos indicadores deben emerger de una discusión y análisis mayor, experto, participativo y democrático, recogiendo las especificidades propias del sistema de educación superior en Chile y de otros países que deseen trabajar con este modelo. 
Tabla 4.

Operacionalización de la variable Resultados.

\begin{tabular}{|c|c|c|}
\hline VARIABLE & CRITERIOS & ELEMENTOS DE EVALUACIÓN \\
\hline \multirow{4}{*}{ Resultados } & $\begin{array}{l}\text { Resultados de } \\
\text { Admisión }\end{array}$ & $\begin{array}{l}\text { Procesos Selección Estudiantes. } \\
\text { Oferta de Programas Formativos. } \\
\text { Demanda de Programas Formativos. }\end{array}$ \\
\hline & $\begin{array}{l}\text { Resultados } \\
\text { Avance } \\
\text { Curricular }\end{array}$ & $\begin{array}{l}\text { Progreso Académico de los Estudiantes. } \\
\text { Niveles de Aprobación, Reprobación y Deserción. } \\
\text { Estudiantes en Proceso de Graduación y/o Titulación. }\end{array}$ \\
\hline & $\begin{array}{l}\text { Resultados } \\
\text { Aprendizaje }\end{array}$ & $\begin{array}{l}\text { Exámenes Nacionales de Conocimientos Profesionales. } \\
\text { Certificación de Competencias Profesionales. }\end{array}$ \\
\hline & $\begin{array}{l}\text { Resultados } \\
\text { Económicos y } \\
\text { Financieros }\end{array}$ & $\begin{array}{l}\text { Formación de Capital Humano Avanzado; Graduados y } \\
\text { Titulados. } \\
\text { Inserción Laboral y Remuneraciones. } \\
\text { Productividad y Remuneraciones. } \\
\text { Gasto Público en Educación Superior. } \\
\text { Aranceles por Carrera para la Educación Superior. } \\
\text { Rentabilidad de la Educación Superior. } \\
\text { Productividad del Gasto Público en Educación Superior. } \\
\text { Situación Económica y Financiera Instituciones de } \\
\text { Educación Superior. }\end{array}$ \\
\hline
\end{tabular}

Tabla 5.

Operacionalización de la variable Información.

\begin{tabular}{|c|c|c|}
\hline VARIABLE & CRITERIOS & ELEMENTOS DE EVALUACIÓN \\
\hline \multirow[b]{3}{*}{ Información } & $\begin{array}{l}\text { Información } \\
\text { Académica }\end{array}$ & $\begin{array}{l}\text { Comunicación de los Requisitos y Puntajes de Admisión } \\
\text { Comunicación de los Estudiantes Seleccionados } \\
\text { Comunicación de la Oferta y Demanda de Programas } \\
\text { Formativos }\end{array}$ \\
\hline & $\begin{array}{l}\text { Información } \\
\text { sobre } \\
\text { Resultados } \\
\text { Aprendizajes }\end{array}$ & $\begin{array}{l}\text { Comunicación Niveles de Aprobación, Reprobación } \\
\text { y Deserción } \\
\text { Comunicación de los Resultados de los Exámenes } \\
\text { Nacionales de Conocimientos y las correspondientes } \\
\text { Certificaciones }\end{array}$ \\
\hline & $\begin{array}{l}\text { Información } \\
\text { sobre los } \\
\text { Resultados } \\
\text { Económicos y } \\
\text { Financieros }\end{array}$ & $\begin{array}{l}\text { Comunicación acerca de la Formación de Capital Humano } \\
\text { Avanzado; Graduados y Titulados. } \\
\text { Comunicación sobre la Inserción Laboral y las } \\
\text { Correspondientes Remuneraciones. } \\
\text { Comunicación de la Productividad y su Correspondencia con } \\
\text { el Nivel de Remuneraciones } \\
\text { Comunicación Sobre el Gasto Público en Educación Superior } \\
\text { Comunicación acerca de los Aranceles y Costos de la } \\
\text { Educación Superior. } \\
\text { Comunicación Sobre la Productividad del Gasto Público en } \\
\text { Educación Superior } \\
\text { Comunicación acerca de la Situación Económica y } \\
\text { Financiera de las Instituciones de Educación Superior }\end{array}$ \\
\hline
\end{tabular}

Tabla 6.

Operacionalización de la variable Consecuencias.

\begin{tabular}{|c|c|c|}
\hline VARIABLE & CRITERIOS & ELEMENTOS DE EVALUACIÓN \\
\hline \multirow{3}{*}{ Consecuencias } & $\begin{array}{l}\text { Económicas y } \\
\text { Financieras }\end{array}$ & $\begin{array}{l}\text { Financiamiento a Entidades Educativas; Asignación de } \\
\text { Fondos Directos e Indirectos y Proyectos de Desarrollo } \\
\text { Institucional. } \\
\text { Financiamiento a Estudiantes; Asignación de Becas, } \\
\text { Créditos Universitarios y Créditos con Aval del Estado. } \\
\text { Financiamiento a Iniciativas de Desarrollo de } \\
\text { Comunidades Educativas. }\end{array}$ \\
\hline & Administrativas & $\begin{array}{l}\text { Acreditación Institucional y/o por Carreras. Publicidad } \\
\text { de Resultados Acreditación. } \\
\text { Autorización de Nuevas Sedes. Decreto de Cierre de } \\
\text { Sedes. } \\
\text { Autorización de Nuevas Carreras. Decreto de Cierre } \\
\text { de Carreras. }\end{array}$ \\
\hline & $\begin{array}{l}\text { Para el Ejercicio } \\
\text { Profesional }\end{array}$ & $\begin{array}{l}\text { Certificados de Habilitación Profesional. Evaluación } \\
\text { Permanente de Competencias Profesionales. } \\
\text { Ejercicio Profesional en el Sector Público. Programas de } \\
\text { Desarrollo para Profesionales de Excelencia. } \\
\text { Programas de Apoyo para la Especialización de } \\
\text { Profesionales Habilitados, dentro del Pais y en el Extranjero }\end{array}$ \\
\hline
\end{tabular}

Tabla 7.

Operacionalización de la variable Comunicación.

\begin{tabular}{l|l|l} 
VARIABLE & CRITERIOS & ELEMENTOS DE EVALUACIÓN \\
& $\begin{array}{l}\text { Comunicación } \\
\text { Institucional a }\end{array}$ & Planificación de la Sociabilización de los Procesos de \\
& Rivel de & Rendición de Cuentas a Nivel de Gobierno Universitario. \\
Gobierno & Planes y Acciones Concretas de Comunicación en \\
Universitario & Ejecución. & Retroalimentación Gobierno Universitario, Academia, \\
Comunicación & & Estudiantes y Estado. \\
& Comunicación & Asimilación del SCT-Chile en la Práctica Educativa. \\
& Académica & Internalización Académica de los Cambios Curriculares. \\
Actividad & Retroalimentación de los Resultados de los \\
& Educativa & Exámenes Nacionales. \\
& Comunicación & Planificación de la sociabilización de los Procesos de \\
Gestión & Rendición de Cuentas a Nivel de Comunidad Educativa. \\
Institucional y & Planes y Acciones Concretas de Comunicación en \\
de Docencia a a & Ejecución. \\
Nivel de & Retroalimentación Rendición de Cuentas \\
Comunidad & a Nivel de Comunidad Educativa. \\
& Educativa &
\end{tabular}




\section{REFERENCIAS}

1. Alexander FK .The Changing Face of Accountability. J High Educ. 2000; 71(4):411-431.

2. Aguerrondo I. La calidad de la educación: ejes para su definición y evaluación [en línea]. Madrid: Organización de Estados Iberoamericanos; 2006 [citado may 2018]. Disponible en: http://campus-oei.org/calidad/aguerrondo.htm

3. Alnoor B. Global Accountabilities Participation, Pluralism, and Public Ethics. Cambridge: Cambridge University Press; 2007.

4. Bonbright D. El rostro cambiante de la rendición de cuentas de las ONG. Seminario Internacional Pregonar con el ejemplo; Sociedad civil y rendición de cuentas; 16 abr 2007; Montevideo, Uruguay. Montevideo: Fundación Kellogg-Instituto de Comunicación y Desarrollo; 2007.

5. Hendel D, Lewis D. Quality Assurance of Higher Education in Transition Countries: Accreditation-Accountability and Assessment. Tert Educ Manag (TEAM). 2005; 11(3):239-258.

6. Universidad de Deusto-Universidad de Groningen. Tuning América Latina. Informe final del proyecto: reflexiones y perspectivas de la educación superior en América Latina. Bilbao: Universidad de Deusto; 2007. 430 p.

7. Shupe D. Productivity, Quality, and Accountability in Higher Education. J Contin High Educ. 1999; 47(1):2-13.

8. Hoecht A. Quality Assurance in UK Higher Education: Issues of Trust, Control, Professional Autonomy and Accountability. High Educ. 2006; 51(4):541-563.

9. Lawrence S, Sharma U. Commodification of Education and Academic Labour: Using the Balanced Scorecard in a University Setting. Crit Perspect Accoun. 2002; 13:661-677.

10. Metz T. Accountability in Higher Education: A Comprehensive Analytical Framework. Theor Res Educ. 2011; 9(1):41-58.
11. Brown R, Carpenter C, Collins R, Winkvist-Noble L. Recents developments in informations about programme quality in the UK. Qual High Educ. 2007; 13(2):173-186.

12. Zapata G, Fleet N. Mercado, rendición de cuentas e información pública en educación superior. Estud Pedagóg. 2012; 38(2):259-276.

13. Huisman J, Currier J. Accountability in Higher Education: Bridge over Troubled Water?. Rev High Educ. 2004: 48:529-551.

14. Conner T, Rabovsky M. Accountability, Affordability, Access: A Review of the Recent Trends in Higher Education Policy Research. Policy Stud J. 2011; 39(1):93-112.

15. National Governors Association Chair's Initiative. Complete to Compete: From Information to Action; Systems Revamping Higher Education Accountability Systems 2010-2011. Washington: NGA Center; 2011.

16. McMeekin R. Hacia una comprensión de la accountability educativa y cómo puede aplicarse en los paises de América Latina. En: Corvalán J, McMeekin $\mathrm{R}$, editores. Accountability educacional; posibilidades y desafios para América Latina a partir de la experiencia internacional CIDE-PREAL [en línea]. Santiago de Chile: PREAL-CIDE; 2006 [citado may 2018]. p. 19-49. Disponible en: http://www.brunner.cl/?p=562

17. Chile Calidad. Premio nacional a la calidad, hacia una gestión de excelencia. Santiago de Chile: Centro Nacional de Productividad y Calidad; 2004. 36 p.

18. Mujica C, Prieto JC. Sistema de créditos transferibles y carga de trabajo de los estudiantes en las universidades del Consejo de Rectores. Rev Calidad Educ. 2007; 26(jul):293-306.

19. Riquelme GM. Estudio de la accountability de la educación superior en Chile, entre los años 1990-2009 [tesis] [en línea]. Málaga: Universidad de Málaga; 2014 [citado may 2018]. Disponible en: http://hdl.handle. net/10630/8275 